\title{
DENSIFICATION AS THE ONLY MECHANISM AT STAKE DURING INDENTATION OF SILICA GLASS?
}

\author{
Vincent Keryvin $^{1,2, \mathrm{a}}$, Solene Gicquel ${ }^{2, \mathrm{~b}}$, Ludovic Charleux ${ }^{2,3, \mathrm{c}}$, Jean-Pierre \\ Guin $^{2, \mathrm{~d}}$, Mariette Nivard ${ }^{2, \mathrm{e}}$ and Jean-Christophe Sanglebœuf ${ }^{2, \mathrm{f}}$ \\ ${ }^{1}$ Univ. Bretagne-Sud, EA 4250, LIMATB, F-56100 Lorient, France \\ ${ }^{2}$ Univ. Rennes 1, ERL CNRS 6274, LARMAUR, F-35000 Rennes, France \\ ${ }^{3}$ Univ. Savoie, EA 4114, SYMME, F-74900 Annecy-Le-Vieux, France \\ avincent.keryvin@univ-ubs.fr, bsolene.gicquel@edf.fr, cludovic.charleux@univ-savoie.fr, \\ djean-pierre.guin@univ-rennes1.fr, ${ }^{e}$ mariette.nivard@univ-rennes1.fr, \\ fjean-christophe.sangleboeuf@univ-rennes1.fr
}

Keywords: Indentation; Glass; Densification; Plasticity; Imprint; Modeling; Finite-Element Analysis; Fused quartz

\begin{abstract}
Silica glass is known to exhibit permanent changes in density under very high pressures. These changes may reach $21 \%$. The sharp indentation test develops pressures underneath the indenter that trigger densification. Recently, we have proposed a constitutive modeling of the pressure-induced process accounting for its salient features: densification threshold, hardening, saturation of densification and permanent increase in elastic moduli. We examine in this paper the possibility that densification could be the only mechanism for creating an imprint by indentation. We consider different models with growing complexity that we implement in a finite element software. Results indicate that the combination of shear and pressure as a driving force to densification may account for the mechanical response of the indentation test as well as the presence of densified zone underneath the imprint.
\end{abstract}

\section{Introduction}

The indentation or the scratching of a glass plate leads to the formation of different cracking systems $[1,2]$. However, for low loads, there exists a regime for which no cracks develop, so that the plasticity of glass is the only responsible for the creation of the imprint. Being amorphous, these materials do not have long range order and thus no crystalline defects such as dislocations that are the plasticity carriers in crystalline materials. The deformation underlying the creation of an imprint in oxide glasses has long been debated these last 40 years $[3,4,5]$. Silicate glasses exhibit permanent changes in volume and density under high hydrostatic pressures. As an example, the density of a- $\mathrm{SiO}_{2}$ (amorphous silica) can be increased by up to $21 \%$ and that of window glass by $6 \%$ for pressures around $25 \mathrm{GPa}$ [6, 7]. Different mechanical models have been proposed these last thirty years $[3,8,9,10]$. The two more recent ones [11, 12] challenged the mechanical response of nano-indentation tests on fused silica with Finite Element Analyses. They found a very good agreement between the experimental load-displacement curve and the numerical simulation, even if their constitutive equations differ quite drastically. They nevertheless consider that the combination of shear and pressure trigger both densification and isochoric plastic strains, as made also on metallic glasses or polymeric glasses to model the indentation response accounting for shear plasticity and cavitation $[13,14]$.

Recently, a constitutive model has been developed to account for the mechanical behaviour of silica glass under hydrostatic pressure, i.e. the permanent changes in density caused by pressure [15]. This pressure-induced densification (PID) is an improvement of previous modellings [11, 12] with considerable impact on the description of the pressure-volume changes of fused silica for pressures lower than $25 \mathrm{GPa}$, taken from very recent experimental data $[16,17]$. In this paper, we examine the 
possibility that densification could be the only dissipative mechanism responsible for the creation of an imprint during the indentation process in silica glass, which is consistent with the experimental observations of Kurkjian et al. [18]. This work is done at room temperature, far from the glass transition, for which shear plasticity is known to be the mechanism at stake, for instance during indentation creep [19].

\section{Experimental details}

Indentation experiments were carried out on a fused quartz sample, which is the calibration standard sample for the Hysitron TI950 apparatus used in this study. The tip is a modified Berkovich diamond pyramid. For each test, the load, $\mathrm{P}$, is increased up to $10 \mathrm{mN}$ with a constant loading rate of $5 \times 10^{-5}$ $\mathrm{N} / \mathrm{s}$. The load is then held for $10 \mathrm{~s}$ and released with a constant unloading rate of $-10^{-4} \mathrm{~N} / \mathrm{s}$. The depth, $h$, is monitored. A truncated tip defect length, $h_{0}$, has been calculated by plotting the square root of $\mathrm{P}$ vs. h. This curve should be linear with a perfect tip. It is not the case for low depths below $50 \mathrm{~nm}$. Thus $\mathrm{h}_{0}$ is calculated by taking the intercept of a linear fit of this curve for high values of $\mathrm{h}$ with the $\mathrm{x}$-axis. $\mathrm{h}_{0}$ is found to be $20 \mathrm{~nm}$.

\section{Numerical procedures}

Finite element modeling (FEM) simulations of the indentation process are performed using a twodimensional axisymmetrical model. The sample is meshed with 3316 four-noded quadrilateral elements. The indenter is considered as a perfect cone exhibiting an half-angle $\Psi=70.29^{\circ}$ to match the theoretical area function of the modified Berkovich indenter. The load on the indenter, $\mathrm{P}$, is controlled and the displacement of its tip, $\mathrm{h}$, is recorded. The dimensions of the mesh are chosen to minimize the effect of the far-field boundary conditions. The typical ratio of the maximum contact radius and the sample size is about $2 \times 10^{3}$. The problem is solved using the commercial software ABAQUS (version 6.10). The indenter material is assumed to be isotropic, linear elastic (Poisson's ratio of 0.07 and Young's modulus of $1100 \mathrm{GPa}$ ). The contact between the indenter and the sample's surface is taken as frictionless. The different constitutive equations have been implemented in the FEM software using a UMAT subroutine. The geometrical non linearities are taken into account using a finite strain framework. To allow a comparison between experiments with an imperfect tip and numerical simulations made with a perfect tip, the experimental displacement has been shifted by $\mathrm{h}_{0}$ to the right. Indeed, by comparing the results of two numerical simulations, one with a perfect tip, the other one with a blunted tip truncated by $\mathrm{h}_{0}$ vis-a-vis a perfect tip, it is found that P-h curves match provided: (i) that the results from the blunted tip simulation are shifted by $\mathrm{h}_{0}$ and (ii) the comparison is made for depths higher than 2-3 times $\mathrm{h}_{0}$.

FEM simulations are also performed on a single cubic element with eight nodes with an imposed pressure of $25 \mathrm{GPa}$ then down to zero to describe the hydrostatic loading with very high pressures.

All constitutive models in this paper are strain-rate and time-independent.

\section{Pressure-induced densification (PID)}

Recently, we have proposed a constitutive model accounting for the different mechanisms in silica glass under pure hydrostatic compression [15]. The salient features of this model are presented here and are schematised on Figure 1. Below a threshold pressure (further referred to as $\mathrm{P}_{0}$, which is $\sim 3$ $\mathrm{GPa}$ ) the behaviour is purely elastic. Densification, $\xi^{p}$, is defined as the opposite of the trace of the plastic strain tensor ${\underset{\sim}{\epsilon}}^{p}$. Accounting for finite strains, it is linked to the permanent changes in density, $\Delta \rho$, via:

$$
\xi^{p}=-\operatorname{tr} \epsilon_{\sim}^{p}=-\ln \left(1+\frac{\Delta \rho}{\rho_{0}}\right)
$$


where $\rho_{0}$ is the initial density (2.20). Densification increases with applied pressure, from $\mathrm{P}_{0}$, in a non linear way, up to a plateau value (saturation of $\sim 0.196$, when accounting for finite strains) for a pressure $\mathrm{P}_{1}$ of $\sim 25 \mathrm{GPa}$. The behaviour is then once again elastic until changes in the medium range order occur (for pressures above $25 \mathrm{GPa}$ ). The model accounts also for the changes in elastic moduli with densification. For example the bulk modulus will double from the pristine state to the fully densified one (from 35.5 to $73.3 \mathrm{GPa}$ ). The parameters of this model have been identified from different ex situ experiments (after decompression). One challenge for this model is to correctly describe the in situ response for the hydrostatic compression test. Newly available data made it possible to have this reference experiment $[16,17]$. Figure 1 is a comparison between these experiments and the numerical simulation with our model, which is very good in contrast to previous modellings as discussed in [15]. The constitutive equations and the values of all model parameters are not presented in details here for reasons of space and clarity; they can be found in [15].

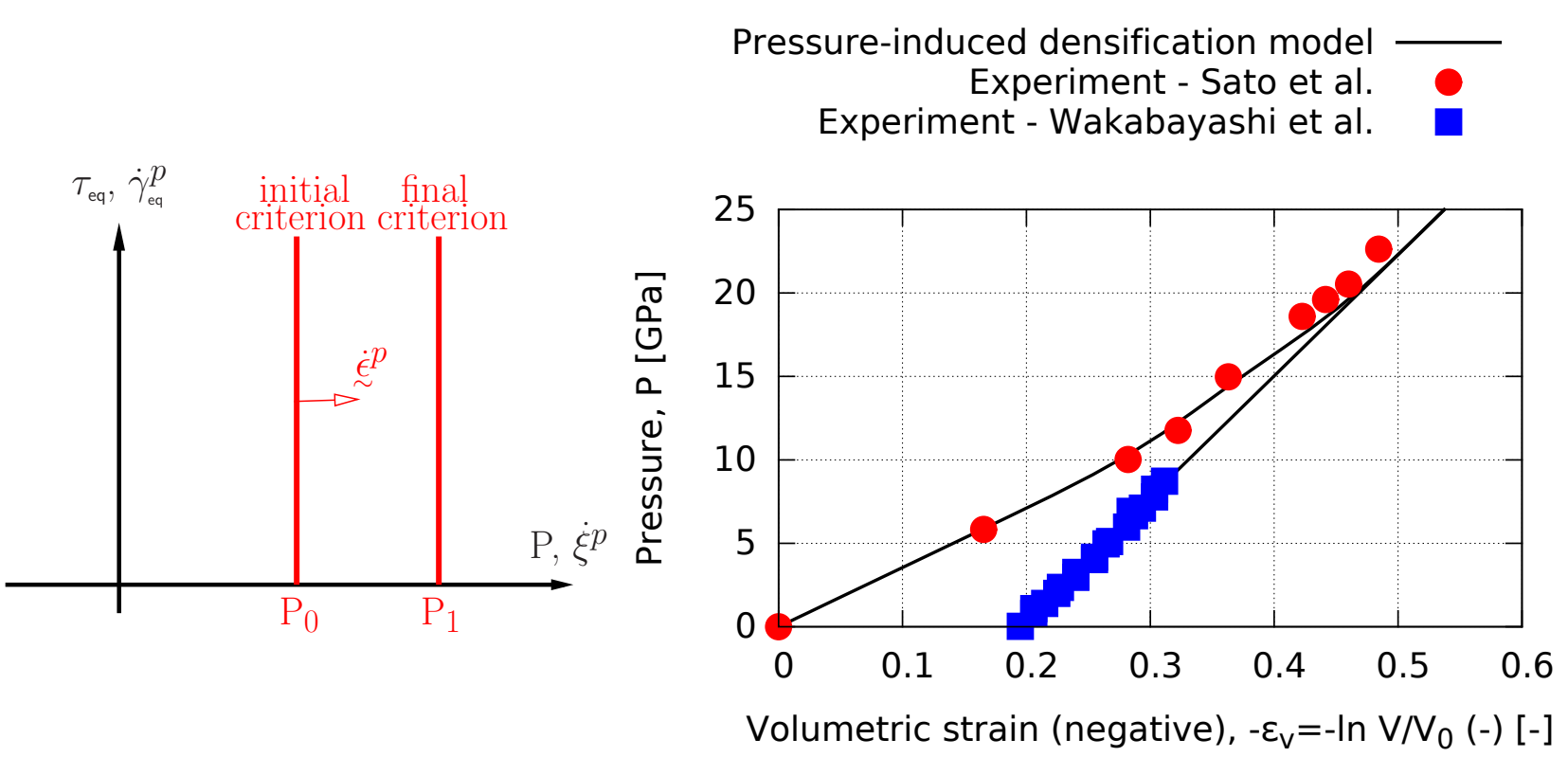

Fig. 1: (Left) Description of the pressure-induced densification model in the shear $\left(\tau_{\mathrm{eq}}\right)$ - pressure (P) plane: the plastic strain rate ${\underset{\sim}{\dot{\epsilon}}}^{p}$ is also superimposed in the isochoric plastic strain rate $\left(\dot{\gamma}_{\text {eq }}^{p}\right)$ vs densification rate $\left(\dot{\xi}^{p}\right)$ plane. $\left(\dot{\xi}^{p}=-\operatorname{tr}{\underset{\sim}{\dot{\epsilon}}}^{p} ; \tau_{\text {eq }}=\sqrt{1 / 2 \operatorname{tr}(\underset{\sim}{S} \cdot \underset{\sim}{S})}, \underset{\sim}{S}\right.$ is the deviatoric part of the Cauchy stress tensor; $\dot{\gamma}_{\text {eq }}^{p}=\sqrt{2 \operatorname{tr}\left(\dot{\sim}^{p} \cdot \dot{\sim}^{p}\right)},{\underset{\sim}{\dot{\gamma}}}^{p}$ is the deviatoric part of ${\underset{\sim}{\dot{\epsilon}}}^{p}$.) (Right) Mechanical response of the hydrostatic compression test on silica glass (pressure vs. volumetric strain), where $V$ and $V_{0}$ denote the actual and initial volumes, respectively. Experimental data: pristine sample (circle symbols) from Sato and Funamori [16] and fully densified sample (square symbols) from Wakabayashi et al. [17]. Comparison with numerical simulations with the pressure-induced densification model from [15].

\section{Indentation behaviour: pressure-induced densification}

We examine now the possibility that pressure-induced densification could be the sole mechanism during an indentation test responsible for the creation of an imprint.

We can notice, in Figure 2, that the numerical simulation with the PID model is quasi-elastic: the load and unload steps are very similar. Therefore, the imprint is quasi absent while densification fields develop underneath the indenter gradually from below the indenter tip, where the saturation in densification is reached, to the elastic containing field.

We can conclude from this Section that there is some pressure-induced densification during the indentation process. However other mechanisms are required to explain the creation of an imprint. 


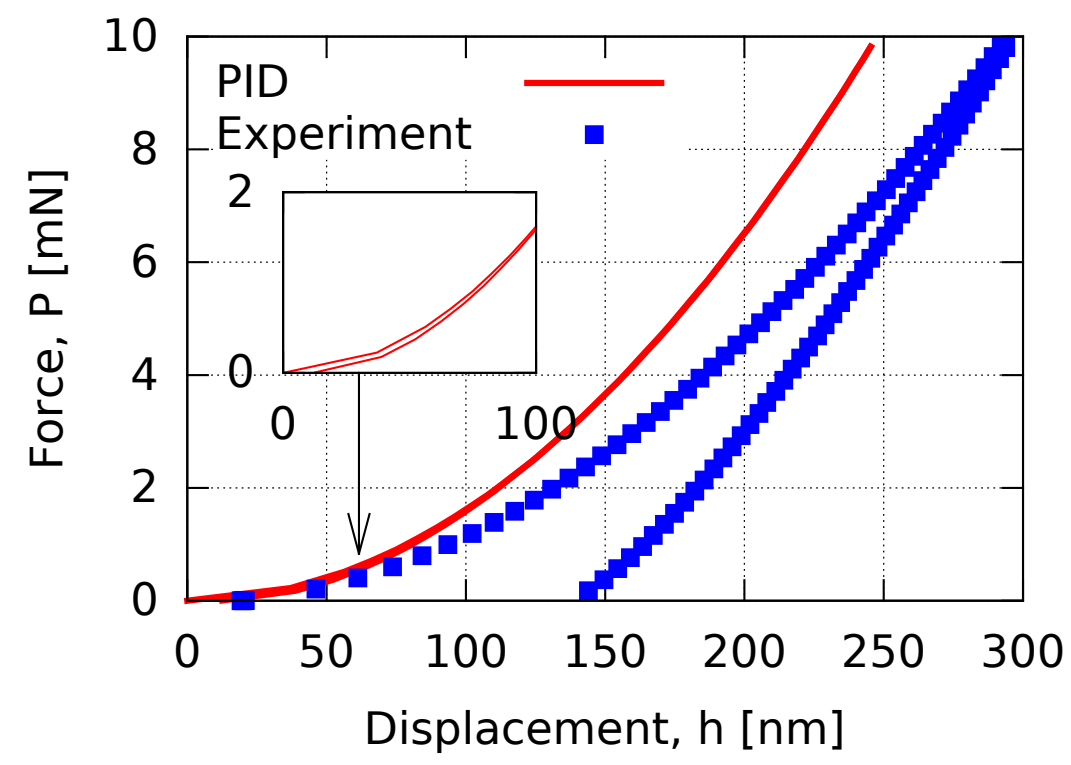

Fig. 2: FEM axisymmetric indentation with the pressure-induced densification model (PID, see Fig. 1). Comparison with a Berkovich nano-indentation test on fused silica; the inset indicates that the simulated behaviour is not reversible.

\section{Indentation behaviour: pressure-induced densification and shear-induced isochoric plastic flow}

Let us examine now the possibility that, in addition to PID, isochoric plastic strains develop, triggered by the sole shear stresses and without any strain hardening. This model is presented Figure 3. It is therefore composed of PID and a von Mises material (perfect $\mathrm{J}_{2}$-plasticity).

By decreasing the shear strength $\mathrm{k}$, the numerical prediction gets softer with respect to the sole PID model. For a shear strength of $\sim 4 \mathrm{GPa}$ the numerical prediction agrees well with the experimental data on fused quartz in terms of residual depth but the overall response is not adequately described. For a value of $2 \mathrm{GPa}$ the loading part of the curve matches quite well with experiments (a value slightly above $2 \mathrm{GPa}$ would do it better) but the unloading curve is far from being correctly descrybed.

The densification field underneath the imprint is represented in Figure $4^{4}$. The maximum level of densification is 0.01 . Even if densification occurs, it is too weak to have an influence on the loaddisplacement curve. Compared to the densification field for the PID model, shear-plasticity "eats" or "swallows" densification. In other words, the response to this model is almost the same as a single von Mises material and therefore can not predict the densification levels experimentally observed, for instance by Raman spectroscopy [20] or deduced by a thermal annealings based technique [21].

\section{Indentation behaviour: pressure and shear - induced densification}

We eventually examine the possibility that densification is the only mechanism at stake during indentation, which is consistent with the experimental observations of Kurkjian et al. [18]. Yet, in contrast to PID, we assume that shear plays a role in triggering the densification process, which is in agreement with recent molecular dynamics studies [22].

The driving force for densification is taken as a combination of shear and pressure. A first possibility consists in a linear combination. We moreover assume that densification will not occur for positive mean stresses (or alternatively negative pressures). We therefore use a von Mises material in this case. The model is represented in the shear-pressure plane in Figure 5. The results presented in Figure 6 indicate clearly that such a model is able to match relatively well the experimental load-displacement curve of fused quartz, for a shear strength value of $\sim 4 \mathrm{GPa}$.

\footnotetext{
${ }^{4}$ Let us highlight that due to the principle of self similarity no scale bar will be given in this kind of figure.
} 
The densification field underneath the imprint is represented in Figure 6 . The densified zone is almost fully saturated (densification value close to the plateau value of 0.196) and there exists a steep transition between the densified zone and the elastic zone. This is in sharp contrast to the results of Raman spectroscopy [20] for which there is a gradual decrease of the densification level from below the tip to the elastic zone. Yet, it has been recently suggested that such a numerical result could be meaningful. Indeed, Niu et al. used a chemical dissolution technique to study densification in oxide glasses including fused quartz [23]. They found a very steep transition zone between the fully densified region and the non densified zone.
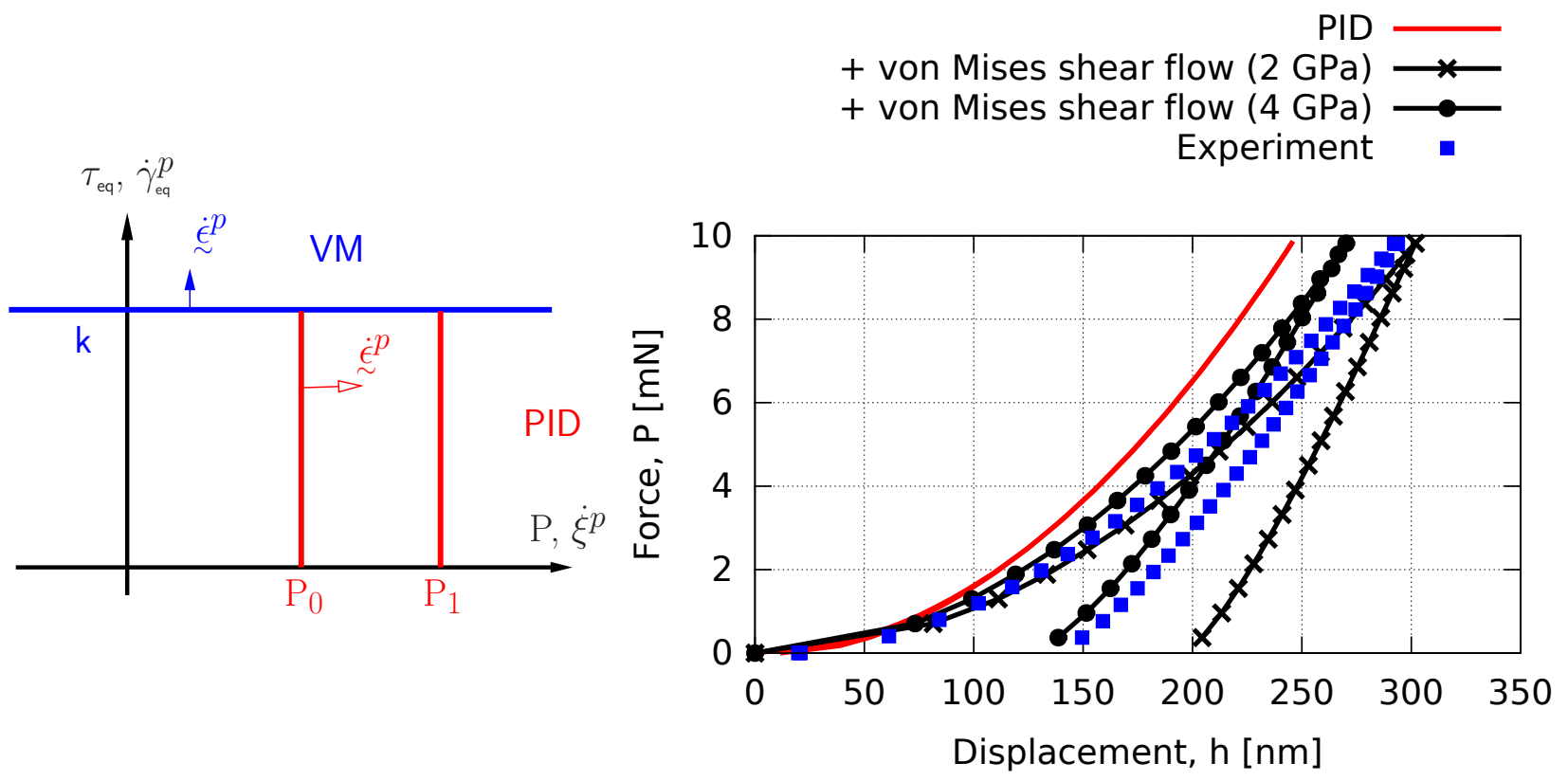

Fig. 3: FEM axisymmetric indentation with the model described on the left side (see Fig. 1 for a description of such a diagram): PID and isochoric shear strains triggered by shear stresses. Comparison with a Berkovich nano-indentation on fused silica (right) with different values of the shear strength $\mathrm{k}$.

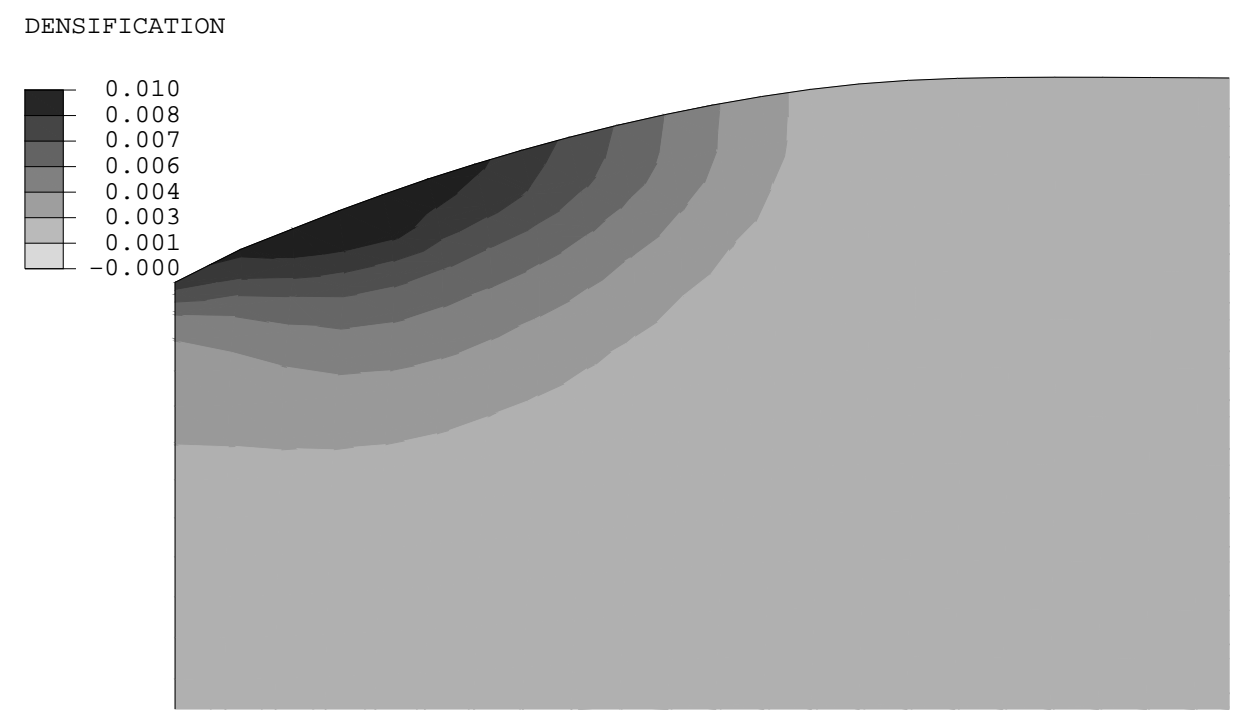

Fig. 4: Densification field underneath the imprint as predicted by FEM with the model described in Fig. 3 for a shear strength of $4 \mathrm{GPa}$. The maximal level of densification is lower than 0.01 , thus far from the saturation value of 0.196 . 



Fig. 5: FEM axisymmetric indentation with the model described on the left side (see Fig. 1 for a description of such a diagram): densification triggered by a linear combination of shear and pressure (for positive pressures) and a von Mises material for positive mean stresses. Comparison with a Berkovich nano-indentation test on fused silica (right) with different values of the shear strength $\mathrm{k}$.

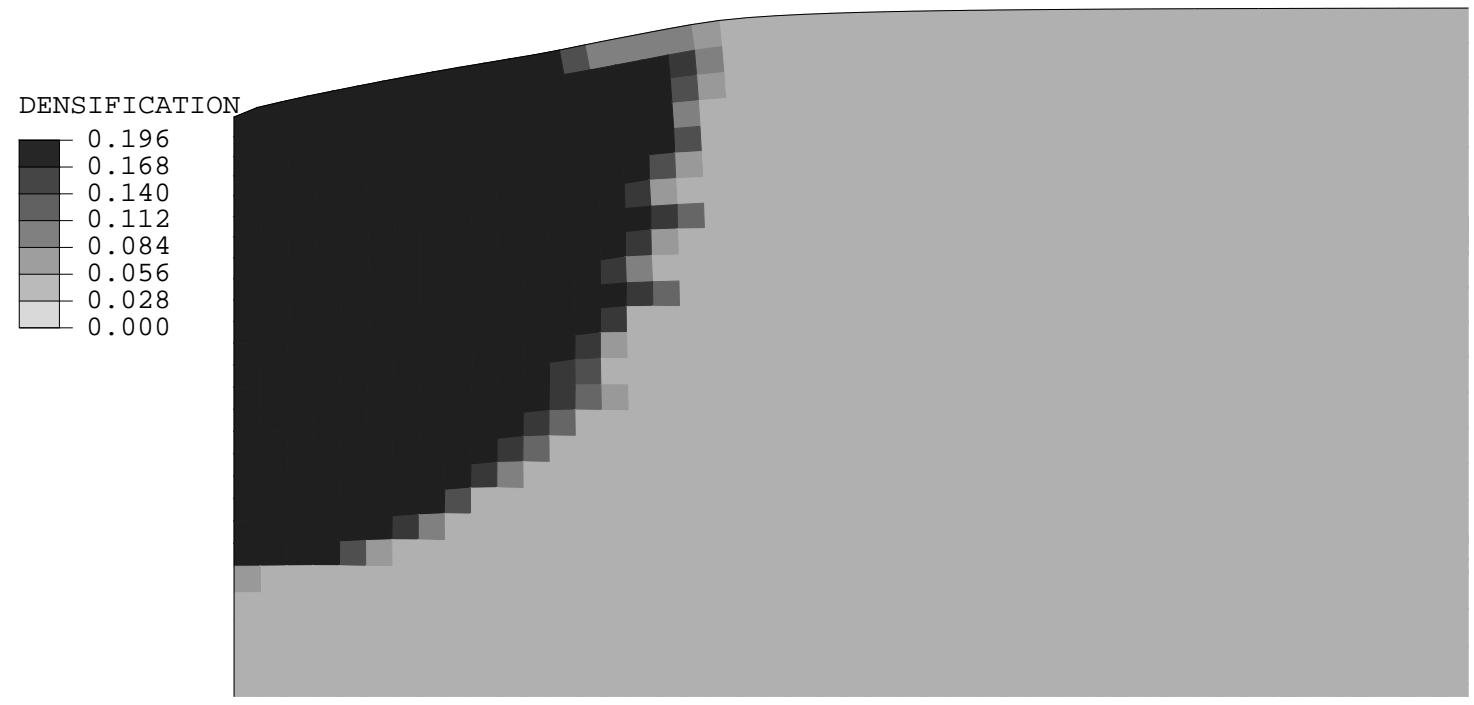

Fig. 6: Densification field underneath the imprint as predicted by FEM with the model described in Fig. 5 for a shear strength of $4 \mathrm{GPa}$ (averaged field on each element). The black zone (upper left and left) corresponds to the zone for which the saturation of densification is reached. One can notice its boundaries (squares with different shades of grey). The light grey zone (bottom right and right) corresponds to the elastic zone. 


\section{Summary and prospects}

In this paper, we have examined the possibility that the sole densification mechanism could be responsible for the mechanical response of the indentation process in fused quartz. Different constitutive models with growing complexity have been challenged to experimental nano-indentation data. They have been implemented in a finite element software by using a material subroutine. Results indicate that neither a pressure-induced densification (PID) nor an uncoupled model with PID and $\mathrm{J}_{2}$ perfect shear plasticity (von Mises material) can account for the mechanical response of the test (the loaddisplacement curve) and the existence of densified zones underneath the indenter. At the contrary, a simple model coupling (for positive pressures) shear and pressure as driving forces for densification can predict both. This possibility and its consequences (a densified zone with a steep transition between the saturated zone and the elastic zone) must now be challenged to other experimental evidence and to molecular dynamics simulations.

\section{Acknowledgements}

Financial support was provided by the Brittany region (ARED 5382 CARAMEL), the French Ministry for Higher Education and Research (PhD grant) and the European University of Brittany (EPT COMPDYNVERRE). They are gratefully acknowledged. VK would like to thank Prof. Philippe Pilvin (University of South-Brittany) for fruitful discussion.

\section{References}

[1] A. Arora, D. B. Marshall, B. R. Lawn, M. V. Swain, Indentation deformation/fracture of normal and anomalous glasses, J. Non. Cryst. Solids 31 (1979) 415-428.

[2] V. le Houérou, J.-C. Sangleboeuf, S. Dériano, T. Rouxel, G. Duisit, Surface damage of sodalime-silica glasses: indentation scratch behavior, J. Non. Cryst. Solids 316 (2003) 54-63.

[3] D. M. Marsh, Plastic Flow and Fracture of Glass, Proc. R. Soc. A Math. Phys. Eng. Sci. 282 (1964) 33-43.

[4] F. M. Ernsberger, Role of Densification in Deformation of Glasses Under Point Loading, J. Am. Ceram. Soc. 51 (1968) 545-547.

[5] K. Peter, Densification and flow phenomena of glass in indentation experiments, J. Non. Cryst. Solids 5 (1970) 103-115.

[6] H. Ji, V. Keryvin, T. Rouxel, T. Hammouda, Densification of window glass under very high pressure and its relevance to Vickers indentation, Scr. Mater. 55 (2006) 1159-1162.

[7] T. Rouxel, H. Ji, V. Keryvin, T. Hammouda, S. Yoshida, Poisson's Ratio and the Glass Network Topology - Relevance to High Pressure Densification and Indentation Behavior, Adv. Mater. Res. 39-40 (2008) 137-146.

[8] M. Imaoka, I. Yasui, Finite element analysis of indentation on glass, J. Non. Cryst. Solids 22 (1976) 315-329.

[9] I. Yasui, M. Imaoka, Finite element analysis of identation on glass(II), J. Non. Cryst. Solids 50 (1982) 219-232.

[10] J. C. Lambropoulos, S. Xu, T. Fang, Constitutive Law for the Densification of Fused Silica, with Applications in Polishing and Microgrinding, J. Am. Ceram. Soc. 79 (1996) 1441-1452. 
[11] K. Xin, J. C. Lambropoulos, Densification of Fused Silica : Effects on Nanoindentation, in: A. J. Marker III, E. G. Arthurs (Eds.), Inorg. Opt. Mater. II. SPIE, vol. 4102 (2000) 112-121.

[12] G. Kermouche, E. Barthel, D. Vandembroucq, P. Dubujet, Mechanical modelling of indentation-induced densification in amorphous silica, Acta Mater. 56 (2008) 3222-3228.

[13] V. Keryvin, Indentation as a probe for pressure sensitivity of metallic glasses., J. Phys. Condens. Matter 20 (2008) 114119.

[14] K. E. Prasad, V. Keryvin, U. Ramamurty, Pressure sensitive flow and constraint factor in amorphous materials below glass transition, J. Mater. Res. 24 (2009) 865-872.

[15] V. Keryvin, J.-X. Meng, S. Gicquel, J.-P. Guin, L. Charleux, J.-C. Sangleboeuf, P. Pilvin, T. Rouxel, G. Le Quilliec, Constitutive modeling of the densification process in silica glass under hydrostatic compression, Acta Mater. 62 (2014) 250-257.

[16] T. Sato, N. Funamori, Sixfold-Coordinated Amorphous Polymorph of SiO2 under High Pressure, Phys. Rev. Lett. 101 (2008) 255502.

[17] D. Wakabayashi, N. Funamori, T. Sato, T. Taniguchi, Compression behavior of densified SiO2 glass, Phys. Rev. B 84 (2011) 144103.

[18] C. R. Kurkjian, G. W. Kammlott, M. M. Chaudhri, Indentation Behavior of Soda-Lime Silica Glass, Fused Silica, and Single-Crystal Quartz at Liquid Nitrogen Temperature, J. Am. Ceram. Soc. 78 (1995) 737-744.

[19] C. Bernard, V. Keryvin, J.-C. Sangleoeuf, T. Rouxel, Indentation creep of window glass around glass transition, Mech. Mater. 42 (2010) 196-206.

[20] A. Perriot, D. Vandembroucq, E. Barthel, V. Martinez, L. Grosvalet, C. Martinet, B. Champagnon, Raman Microspectroscopic Characterization of Amorphous Silica Plastic Behavior, J. Am. Ceram. Soc. 89 (2006) 596-601.

[21] S. Yoshida, J.-C. Sangleboeuf, T. Rouxel, Quantitative evaluation of indentation-induced densification in glass, J. Mater. Res. 20 (2005) 3404-3412.

[22] B. Mantisi, A. Tanguy, G. Kermouche, E. Barthel, Atomistic response of a model silica glass under shear and pressure, Eur. Phys. J. B 85 (2012) 304.

[23] Y.-F. Niu, K. Han, J.-P. Guin, Locally enhanced dissolution rate as a probe for nanocontactinduced densification in oxide glasses., Langmuir 28 (2012) 10733-40. 